\title{
Perinatal Risk Factors and Later Social, Thought, and Attention Problems after Perinatal Stroke
}

\author{
Mary J. Harbert, ${ }^{1}$ Micaela Jett, ${ }^{2}$ Mark Appelbaum, ${ }^{3}$ Ruth Nass, ${ }^{4}$ and Doris A. Trauner ${ }^{5}$ \\ ${ }^{1}$ Department of Neurosciences, University of California San Diego School of Medicine and Rady Children's Hospital-San Diego, \\ La Jolla, CA 92093, USA \\ 2 47th Medical Group, 47 MDOS/SGOBP, Laughlin AFB, Dcl Rio, TX 78843, USA \\ ${ }^{3}$ Department of Psychology, University of California San Diego, La Jolla, CA 92093, USA \\ ${ }^{4}$ Departments of Neurology, Psychiatry and Pediatrics, NYU Langone Medical Center, New York, NY 10016, USA \\ ${ }^{5}$ Departments of Neurosciences and Pediatrics, University of California San Diego School of Medicine and \\ Rady Children's Hospital-San Diego, 9500 Gilman Drive, La Jolla, CA 92093, USA
}

Correspondence should be addressed to Doris A. Trauner, dtrauner@ucsd.edu

Received 21 January 2012; Revised 18 March 2012; Accepted 19 March 2012

Academic Editor: Halvor Naess

Copyright (C) 2012 Mary J. Harbert et al. This is an open access article distributed under the Creative Commons Attribution License, which permits unrestricted use, distribution, and reproduction in any medium, provided the original work is properly cited.

\begin{abstract}
Objective. Survivors of perinatal stroke may be at risk for behavioral problems. Perinatal risk factors that might increase the likelihood of later behavior problems have not been identified. The goal of this study was to explore whether perinatal factors might contribute to behavior problems after perinatal stroke. Methods. 79 children with unilateral perinatal stroke were studied. Perinatal factors included gender, gestational age, neonatal seizures, instrumented delivery, fetal distress, acute birth problems, birth weight, and time of diagnosis. Subjects with evidence of hypoxic ischemic encephalopathy were excluded. Parents completed the Achenbach Child Behavior Checklist (CBCL) (Achenbach 1985). The CBCL yields T-scores in several symptom scales. We focused on Social, Thought, and Attention Problems scates. Results. Gestational age and the presence of uteroplacental insufficiency were associated with significant differences on the Thought Problems scale; Attention Problems scores approached significance for these variables. Fetal distress, neonatal seizures, or neonatal diagnosis was associated with 25-30\% incidence of clinically significant T-scores on Social, Thought, and Attention Problems scales. Conclusions. Several perinatal factors were associated with a high incidence of social, thought, and behavior problems in children with perinatal stroke. These findings may be useful in anticipatory guidance to parents and physicians caring for these children.
\end{abstract}

\section{Introduction}

Perinatal stroke is surprisingly common, affecting 1 in 2300 to 4000 children per year. The incidence of stroke in neonates is second only to that of the elderly [1]. Perinatal stroke is the primary cause of hemiplegic cerebral palsy. In addition to motor impairment, children with perinatal stroke are at risk for other problems such as epilepsy, learning disorders, cognitive impairment, language delay, and behavioral problems $[2,3]$. A number of studies have reported a higher incidence of behavioral problems, particularly social and attention problems, in children with early focal brain injury $[2,4]$.
Early studies reported that children with hemiparesis or unilateral abnormalities on neurological exam (although without radiological confirmation of a unilateral brain lesion) were found to have more social difficulties than children without hemiparesis [5-7]. Lee et al. found that of 36 children with perinatal stroke, 22\% were later diagnosed with behavioral problems [2]. This study was a retrospective review of stroke cases in the Kaiser Health System and utilized medical records reports to identify the presence of behavioral problems. Almost 1/4 of the patients (8 of 36) were said to have behavior problems that included hyperactivity and poor attention. Both unilateral and bilateral stroke cases were included in this report. A cohort study of 
children with hemiplegic CP found that half had behavioral or psychiatric problems, including hyperactivity, conduct disorders, and oppositional behavior $[8,9]$. The mode of ascertainment in this study (presence of cerebral palsy rather than documentation of a unilateral infarct of perinatal onset) may have led to a heterogeneous subject group, and thus it is difficult to determine how relevant those studies were to the perinatal stroke population. Max et al. investigated attention in children with childhood stroke with onset at different ages [10]. They found that attention was significantly poorer than matched controls and that the younger the age at lesion acquisition, the poorer the attentional ability. Other studies using a very homogeneous group of children with radiographically and historically documented evidence of unilateral perinatal stroke found a surprisingly low incidence of later behavioral problems in that population $[11,12]$. A small percentage were found to have social, thought, and attention problems compared with controls, but the majority of children did not demonstrate such behavioral issues. Talib et al. studied a small group of twin children with perinatal stroke and compared them to their unaffected twin. There was no clear pattern of attentional impairment in the twin with stroke [13].

Although these earlier studies suggest the possibility that children with perinatal stroke have attention and social difficulties, it is not clear from previous studies whether there are specific risk factors within the perinatal stroke population that may predispose to later behavioral difficulties. The goal of the present study was to examine the occurrence of specific perinatal risk factors and their potential association with later social, thought, and attention problems as identified by a parent report, the Child Behavior Checklist.

\section{Methods}

In a large prospective cohort study, 79 children with a diagnosis of perinatal or presumed perinatal stroke were identified. Recruitment for subjects in this cohort was approved by the Institutional Review Board at the University of California San Diego. Subjects were recruited from 1983 to 2006 via referrals from a large catchment area in southern California and also from the New York metropolitan area. Perinatal stroke subjects presented in the neonatal period with seizures and/or encephalopathy. Subjects with presumed perinatal stroke presented after the neonatal period, typically with hemiparesis or epilepsy. All subjects had evidence of a focal unilateral infarct on neuroimaging (either CT or MRI).

Birth information and hospital data from the inpatient stay as a newborn (if available) were obtained for each subject. Variables considered included the presence of neonatal seizures, gestational age, uteroplacental insufficiency (defined by poor maternal weight gain, small-forgestational-age infant, maternal hypertension and/or preeclampsia, maternal chronic disease, maternal smoking, maternal infection, placental event such as thrombus or abruption, or twin-twin transfusion syndrome), presence of fetal distress (defined as nonreassuring fetal heart tracings, need for emergency C-section, need for instrumentation at delivery, presence of meconium, or diagnosis of chorioamnionitis), and use of instrumentation at delivery (assistance via forceps or vacuum). Exclusionary criteria included a history of intrauterine drug exposure, evidence of neonatal hypoxic-ischemic encephalopathy, neonatal meningitis, or bilateral or multifocal lesions on neuroimaging studies. Imaging variables collected included lesion side and lesion severity. Lesion severity was graded on a 1-5 scale in which $1=$ focal ventricular dilation/atrophy seen on $<3$ cuts on CT or MRI; 2 = focal ventricular dilation/atrophy seen on $>3$ cuts on CT or MRI; 3 = focal porencephaly involving one lobe and observed in $<3$ cuts on CT or MRI; $4=$ focal porencephaly involving one lobe and observed on $>3$ cuts on CT or MRI; 5 $=$ porencephaly or atrophy involving multiple lobes.

For each subject, a parent completed the Achenbach Child Behavior CheckList (CBCL), which is a 113-item checklist validated for use in children 4-18 years of age [14]. The parent responses are based on their observations of behaviors in the two months prior to the questionnaire. Each item is scored on a $0-2$ scale, in which $0=$ not true, $1=$ somewhat or sometimes true, and $2=$ very true or often true. Nine Behavior Problem Scales, as well as Internalizing and Externalizing scores and a Total Problem score are derived. T-scores above 70 are considered clinically significant, while scores between 67 and 70 are in the "borderline clinical" range. Based on our previous study indicating that children with perinatal stroke differed from controls only on Social, Thought, and Attention Problems scales [12], we focused our analyses on these problem scales only.

Statistical analyses were performed using IBM SPSS Version 15.0 for Windows (2006 IBM Corporation.) The Pearson correlation was employed for comparisons using categorical variables, and the ANOVA test was used for continuous variables. $P$ values of $\leq .05$ were considered to be statistically significant.

\section{Results}

Seventy-nine cases with focal infarct had parent-completed CBCL evaluations. The mean age of the child at the time of CBCL evaluation was 9.4 years; subjects ranged from 4 to 19 years of age. Fifty of the subjects $(63 \%)$ were male. Fourteen subjects were born preterm ( $<37$ weeks gestation), 52 were term, 9 were postterm ( $>42$ weeks), and 4 had unknown gestational age. Eleven infants were delivered with instrumentation (forceps or vacuum-assisted delivery). Twenty-five subjects (32\%) had a history of fetal distress, and 19 (24\%) had evidence of uteroplacental insufficiency. Twenty-seven of 79 subjects (34\%) presented in the newborn period, and 52 of 79 presented after the newborn period (66\%). Twenty-four subjects (34\%) presented with neonatal seizures. On neuroimaging, 50 subjects $(63 \%)$ had left-sided lesions. Fifty-two patients (66\%) had hemiparesis.

Gestational age and the presence of uteroplacental insufficiency were associated with significant differences on the Thought Problems scale, and Attention Problems scores approached significance for these variables. Fetal distress was associated with higher Attention Scale T-scores approaching 
TABLe 1: $P$ values reflecting perinatal risk factors and elevated CBCL scores.*

\begin{tabular}{lcccc}
\hline & Total score & Social problem score & Thought problem score & Attention problem score \\
\hline Gender & 0.18 & 0.74 & 0.52 & 0.99 \\
Neonatal seizures & 0.36 & 0.70 & 0.49 & 0.06 \\
Gestational age & 0.20 & 0.43 & $\mathbf{0 . 0 4}$ & 0.07 \\
Uteroplacental insufficiency & 0.13 & 0.93 & 0.05 & 0.08 \\
Fetal distress & 0.55 & 0.97 & 0.88 & 0.06 \\
Instrumentation & 0.24 & 0.76 & 0.31 & 0.53 \\
Lesion side & 0.23 & 0.33 & 0.43 & 0.49 \\
Lesion severity & 0.98 & 0.54 & 0.19 & 0.23 \\
Hemiparesis & 0.86 & 0.18 & 0.91 & 0.48 \\
Presentation in neonatal period & 0.47 & 0.84 & 0.25 & 0.08 \\
Age at testing & 0.70 & 0.48 & 0.49 & \\
\hline
\end{tabular}

$* P$ values obtained using one-way ANOVA.

traditional levels of statistical significance $(-4.56,95 \% \mathrm{CI}$ $2.41, P=0.06)$, and the presence of neonatal seizures was similarly associated with higher Attention Scale scores $(-4.47,95 \%$ CI $2.36, P=0.06)$. Subjects diagnosed in the neonatal period had higher Attention Scale scores than subjects presenting after the newborn period, and results approached traditional levels of statistical significance (4.07, 95\% CI 2.26, $P=0.08$.)

Table 1 displays all relationships between CBCL T-score subsets, perinatal variables, and nonperinatal variables. Some elevated CBCL subscores were correlated with nonperinatal variables. The side of the lesion was not associated with abnormal T-scores. The presence of hemiparesis was not associated with elevated total score or subscores. Age at the time of CBCL testing was also not associated with any elevation in scores.

Although there were no statistically significant differences found between perinatal risk factors and the behavioral measures examined, we qualitatively reviewed all T-scores of 70 or above (clinically significant range of behavior) within each category. Figure 1 shows a pattern in which clinically significant T-score elevations for Social Problems are present in approximately $1 / 3$ of all perinatal stroke infants with fetal distress, neonatal seizures, or a diagnosis in the neonatal period; these children also had a higher percentage of $\mathrm{T}>$ 70 than children without fetal distress or neonatal seizures or whose diagnosis was not made until several months after birth. Similarly, children with a history of fetal distress, neonatal seizures, or neonatal diagnosis had a higher percentage of T-scores $>70$ on Thought and Attention scales than did those children without such a history.

\section{Discussion}

Children with perinatal stroke may be at higher risk for developmental, cognitive, motor, and behavioral problems compared with the general pediatric population. Previous studies have had mixed results, with some finding major behavioral/psychiatric disorders $[8,9,15]$ while others found little evidence of behavioral dysfunction [11, 12]. Many of the previous studies are limited by either small sample size

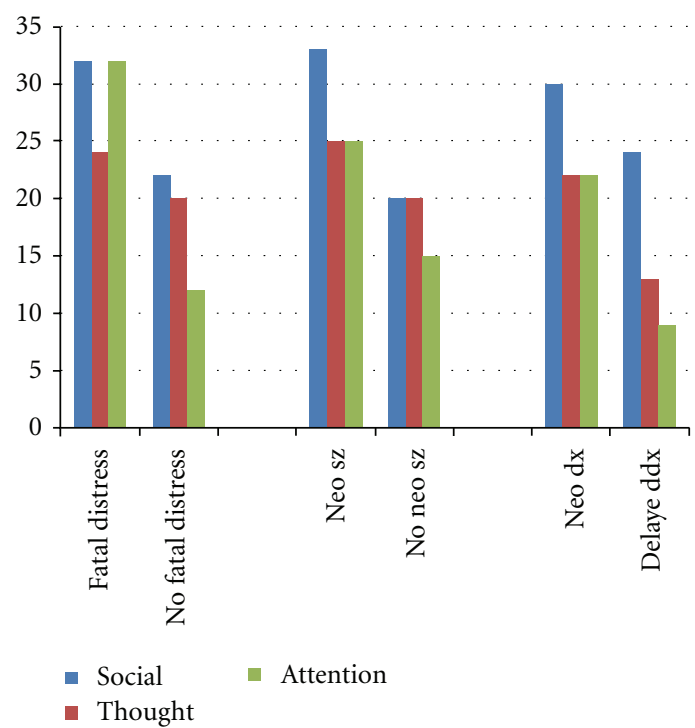

Figure 1: Percent of cases with T-scores $\geq 70$ on Social, Thought, or Attention Problems scales of the CBCL, related to the presence or absence of fetal distress, neonatal seizures, or time of diagnosis.

or utilizing a heterogeneous group of children (hemiplegic without radiological confirmation of lesion, bilateral lesions, mixed etiologies, and/or timing of lesion onset). The current study used a homogeneous group of children with unilateral perinatal stroke and set out to determine whether specific perinatal problems (other than HIE) might place the child at higher risk for later behavior problems. Our results failed to show a significant relationship between perinatal factors and later social, thought, or attention problems as measured by the Child Behavior Checklist. Three specific risk factors, the presence of fetal distress, neonatal seizures, and diagnosis of stroke in the neonatal period, did, however, correspond to higher mean T-scores on the Attention Problems scale, and the results approached statistical significance. Furthermore, a qualitative analysis of the data using a clinically significant T-score of $\geq 70$ found that more than $30 \%$ of children with these risk factors had clinically elevated T-scores on Social 
Problems, and 22-32\% of children with the same risk factors had T-scores $\geq 70$ on the Thought and Attention Problems scales. These percentages are markedly higher than would be expected in a typical pediatric population [12] and indicate that certain perinatal factors may place a child at higher risk for later social and attention problems.

Although a number of studies have looked at perinatal factors that might predict later behavior or social difficulties, we found surprisingly little research on the influence of fetal distress or neonatal seizures on psychosocial function in childhood. Most studies have focused on the influence of birth weight, gestational age, and infant feeding on later psychosocial difficulties [16, 17]. Jacobs and colleagues have conducted several studies of early brain injury of diverse causes and ages and have found that social problems are more likely to be present if the brain injury occurred prior to 2 years of age [18]. We found no evidence for an association between gestational age and later attention or social difficulties in our subject population. The role of other specific perinatal factors, including fetal distress and neonatal seizures, has not been previously examined in detail. One study of long-term outcome after neonatal seizures in "normal" survivors found that over half of the children studied had social adjustment difficulties in their teenage years [19]. In contrast, a study from Canada found no significant group differences on behavioral measures in various groups of "high-risk" children, including those who had neonatal seizures [20]. The risk of neonatal seizures and fetal distress in the perinatal stroke population has not been previously reported, to our knowledge. Though fetal distress and encephalopathy are clinically more likely to be associated with global hypoxic injury in the newborn period, both can be presenting features of perinatal stroke. A significant percentage of neonates presenting with presumed hypoxic encephalopathy are actually later found on imaging to have a focal ischemic stroke [21].

Our study thus provides needed information about the role of specific perinatal factors that may increase the likelihood that a child with perinatal stroke will exhibit social, thought, and/or attention problems during childhood. These children may derive great benefit from early intervention for behavioral problems, such as implementation of individualized education plans, social skills training, individual counseling, and pharmacotherapy.

\section{Acknowledgments}

This research was supported by the National Institutes of Health (P50 NS22343, Center for the Study of the Neurological Basis of Language, D. A. Trauner PI; RO1 NS042584, Neuro-cognitive Outcome after Early Focal Brain Damage, D. A. Trauner PI; RO1 DC06098, Language Development Following Early Focal Brain Injury, D. A. Trauner PI).

\section{References}

[1] J. K. Lynch and K. B. Nelson, "Epidemiology of perinatal stroke," Current Opinion in Pediatrics, vol. 13, no. 6, pp. 499$505,2001$.
[2] J. Lee, L. A. Croen, C. Lindan et al., "Predictors of outcome in perinatal arterial stroke: a population-based study," Annals of Neurology, vol. 58, no. 2, pp. 303-308, 2005.

[3] D. A. Trauner, C. Chase, P. Walker, and B. Wulfeck, "Neurologic profiles of infants and children after perinatal stroke," Pediatric Neurology, vol. 9, no. 5, pp. 383-386, 1993.

[4] J. E. Max, "Effect of side of lesion on neuropsychological performance in childhood stroke," Journal of the International Neuropsychological Society, vol. 10, no. 5, pp. 698-708, 2004.

[5] M. B. Denckla, "The neuropsychology of social-emotional learning disabilities," Archives of Neurology, vol. 40, no. 8, pp. 461-462, 1983.

[6] D. Tranel, L. E. Hall, S. Olson, and N. N. Tranel, "Evidence for a right-hemisphere developmental learning disability," Developmental Neuropsychology, vol. 3, pp. 114-127, 1987.

[7] S. Weintraub and M. Marsel Mesulam, "Developmental learning disabilities of the right hemisphere. Emotional, interpersonal, and cognitive components," Archives of Neurology, vol. 40, no. 8, pp. 463-468, 1983.

[8] R. Goodman and P. Graham, "Psychiatric problems in children with hemiplegia: cross sectional epidemiological survey," British Medical Journal, vol. 312, no. 7038, pp. 1065-1069, 1996.

[9] R. Goodman and C. Yude, "Do unilateral lesions of the developing brain have side-specific psychiatric consequences in childhood?" Laterality, vol. 2, no. 2, pp. 103-115, 1997.

[10] J. E. Max, D. A. Robin, H. G. Taylor et al., "Attention function after childhood stroke," Journal of the International Neuropsychological Society, vol. 10, no. 7, pp. 976-986, 2004.

[11] D. A. Trauner, J. L. Panyard-Davis, and A. O. Ballantyne, "Behavioral differences in school age children after perinatal stroke," Assessment, vol. 3, no. 3, pp. 265-276, 1996.

[12] D. A. Trauner, R. Nass, and A. Ballantyne, "Behavioural profiles of children and adolescents after pre- or perinatal unilateral brain damage," Brain, vol. 124, no. 5, pp. 995-1002, 2001.

[13] T. L. Talib, S. J. Pongonis, L. S. Williams et al., "Neuropsychologic outcomes in a case series of twins discordant for perinatal stroke," Pediatric Neurology, vol. 38, no. 2, pp. 118-125, 2008.

[14] T. M. Achenbach, Assessment and Taxonomy of Child and Adolescent Psychopathology, Sage, Thousand Oaks, Calif, USA, 1985.

[15] R. Goodman, "The longitudinal stability of psychiatric problems in children with hemiplegia," Journal of Child Psychology and Psychiatry and Allied Disciplines, vol. 39, no. 3, pp. 347354, 1998.

[16] W. H. Oddy, M. Robinson, G. E. Kendall, J. Li, S. R. Zubrick, and F. J. Stanley, "Breastfeeding and early child development: a prospective cohort study," Acta Paediatrica, vol. 100, no. 7, pp. 992-999, 2011.

[17] C. Galéra, S. M. Côté, M. P. Bouvard et al., "Early risk factors for hyperactivity-impulsivity and inattention trajectories from age 17 months to 8 years," Archives of General Psychiatry, vol. 68, no. 12, pp. 1267-1275, 2011.

[18] R. Jacobs, A. S. Harvey, and V. Anderson, "Executive function following focal frontal lobe lesions: impact of timing of lesion on outcome," Cortex, vol. 43, no. 6, pp. 792-805, 2007.

[19] C. M. Temple, J. Dennis, R. Carney, and J. Sharich, "Neonatal seizures: long-term outcome and cognitive development among "normal" survivors," Developmental Medicine and Child Neurology, vol. 37, no. 2, pp. 109-118, 1995. 
[20] A. K. F. Li, R. S. Sauve, and D. E. Creighton, "Early indicators of learning problems in high-risk children," Journal of Developmental and Behavioral Pediatrics, vol. 11, no. 1, pp. 1-6, 1990.

[21] J. K. Lynch, D. G. Hirtz, G. DeVeber, and K. B. Nelson, "Report of the National Institute of neurological disorders and stroke workshop on perinatal and childhood stroke," Pediatrics, vol. 109, no. 1, pp. 116-123, 2002. 


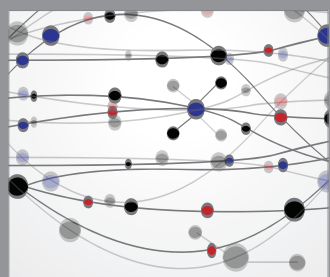

The Scientific World Journal
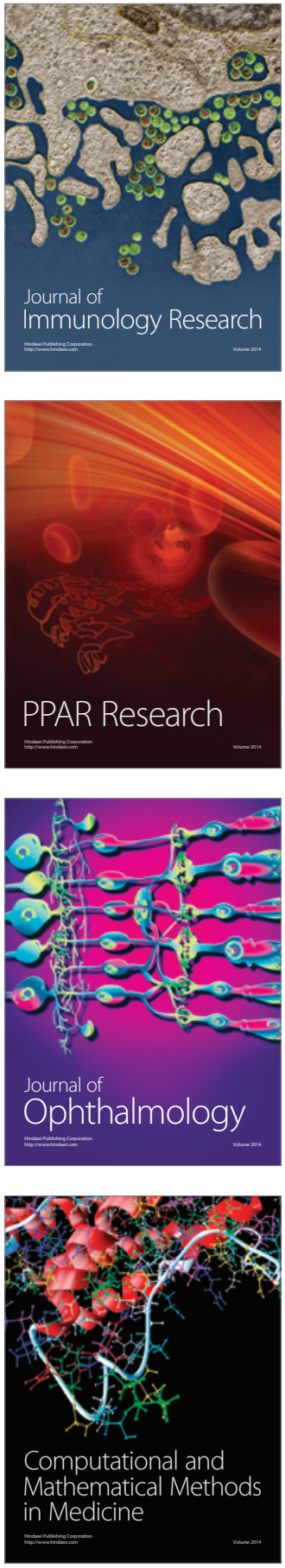

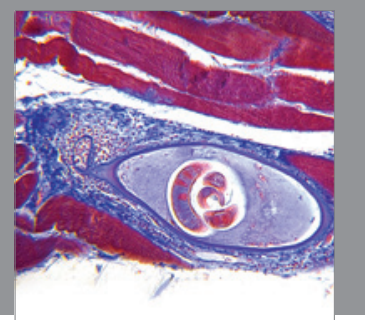

Gastroenterology

Research and Practice
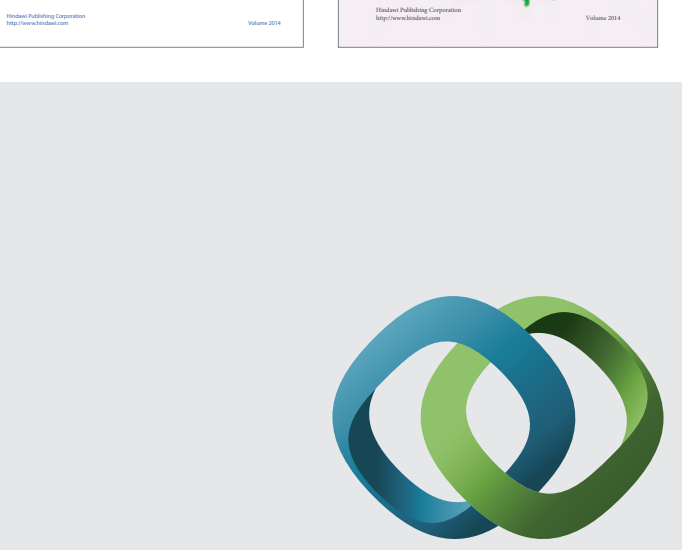

\section{Hindawi}

Submit your manuscripts at

http://www.hindawi.com
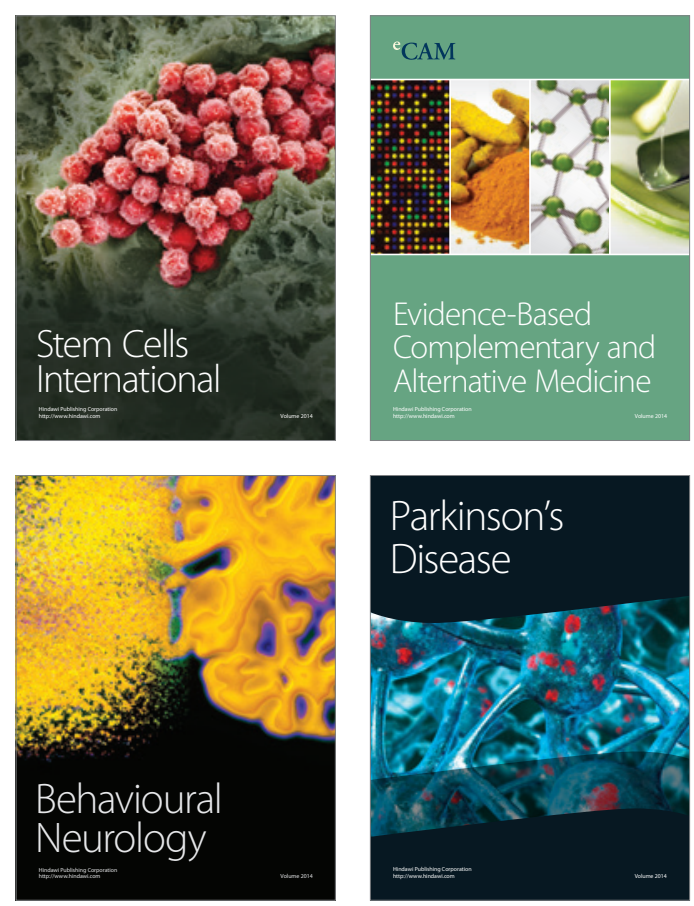

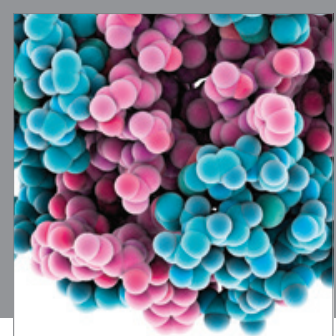

Journal of
Diabetes Research

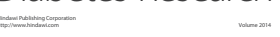

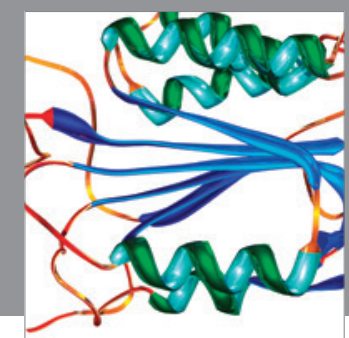

Disease Markers
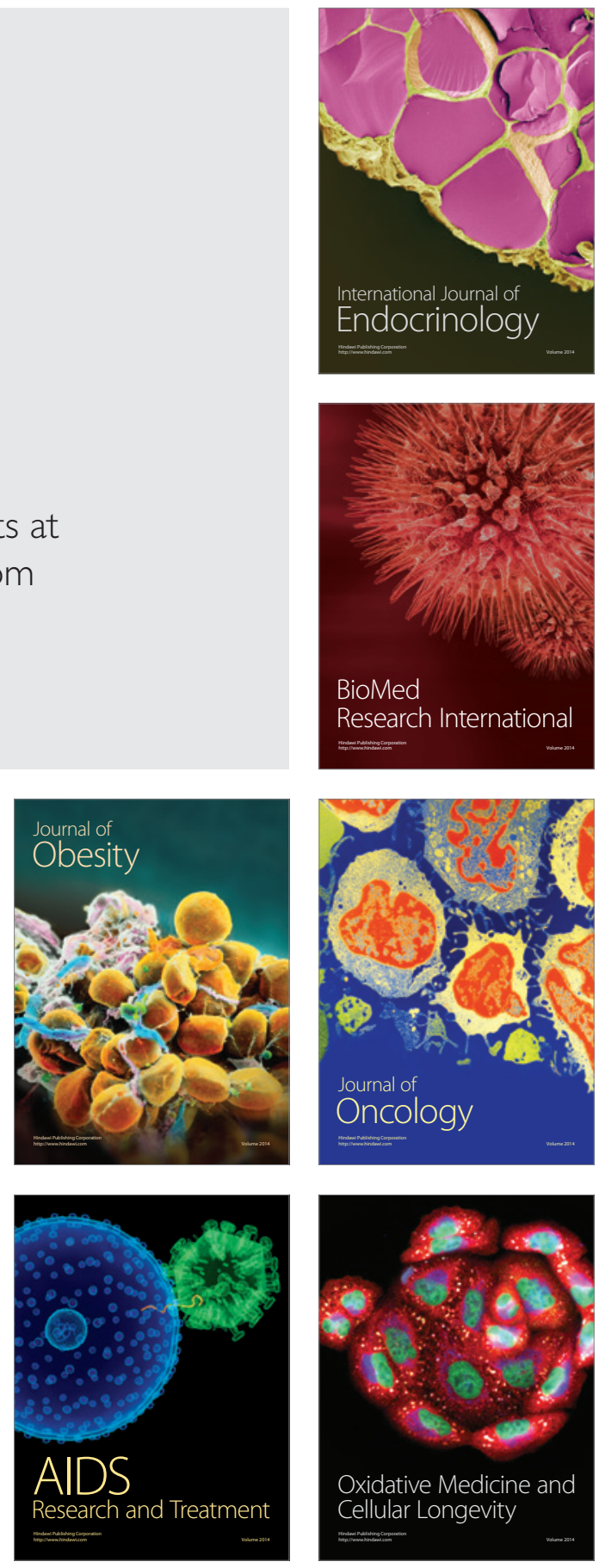